Kuno Weise

BG-UnfallklinikTübingen

\title{
Zugangswahl und Osteosynthesetechnik bei komplexen Azetabulumfrakturen
}

\section{Zusammenfassung}

Komplexe Azetabulumfrakturen sind als knöcherne Verletzungen der Hüftpfanne definiert, welche über eine 1-Pfeiler-Fraktur hinausgehen. Ihre Klassifikation erfolgt vornehmlich nach der Einteilung von Judet und Letournel in einfache und kombinierte Bruchformen. Die AO-Klassifikation versteht unter komplexen Azetabulumfrakturen verschiedene Formen und Kombinationen einer Querfraktur (Typ B) und 2-Pfeiler-Frakturen (Typ C). Ein für alle Frakturen geeigneter Standardzugang steht nicht zur Verfügung. Nahezu alle Frakturen können über den dorsalen Kocher-Langenbeck- oder den ilioinguinalen Zugang (evtl. kombiniert) versorgt werden. Erweiterte Zugänge sind die Ausnahme. Die Rekonstruktion komplexer Azetabulumfrakturen erfolgt nach anatomischer Reposition unter Zuhilfenahme spezieller Instrumentarien sowie mit Schrauben und Rekonstruktionsplatten aus dem Kleinfragmentinstrumentarium.

\section{Schlüsselwörter}

Komplexe Azetabulumfrakturen . Zugangswahl · Osteosynthesetechnik
Momplexe Frakturen des Azetabulums sind knöcherne Läsionen der Hüftgelenkpfanne, welche mehr als 1 Pfeiler betreffen. Diese meist im Rahmen von Rasanztraumen, speziell bei Verkehrsunfällen bzw. bei Stürzen aus größerer Höhe, entstehenden Verletzungen gehören zu den am schwierigsten zu versorgenden Frakturen des Skeletts, da ihre möglichst vollständige Rekonstruktion nur auf der Basis einer umfangreichen Diagnostik, der exakten Planung von der Lagerung über den Zugangsweg bis zur Art der Stabilisierung und durch einen erfahrenen, mit der Anatomie des Beckens und den Möglichkeiten der Osteosynthese vertrauten Chirurgen gelingen kann. Da die Inzidenz komplexer Azetabulumfrakturen insgesamt als nicht sehr hoch anzusehen ist, sind dem Erwerb solch umfangreicher Erfahrungen für den einzelnen Operateur zwangsläufig Grenzen gesetzt. So ist aus einer Multizenterstudie der AG Becken der DGU zusammen mit der Deutschen Sektion der AOI [2] zu ersehen, dass während einer Laufzeit von 3 Jahren zwischen 1991 und 1993 in den 10 beteiligten Kliniken insgesamt 537 Azetabulumfrakturen konservativ oder operativ behandelt wurden. Nahezu 1/3 dieser Verletzungen wurden ohne Operation ausbehandelt, sodass pro Klinik und Jahr durchschnittlich nur 12,5 Fälle zur Operation anstanden. Unter Berücksichtigung des Umstandes, dass es sich bei diesen operativen Eingriffen teilweise um die Versorgung einfacher hinterer Wand- und Pfei-

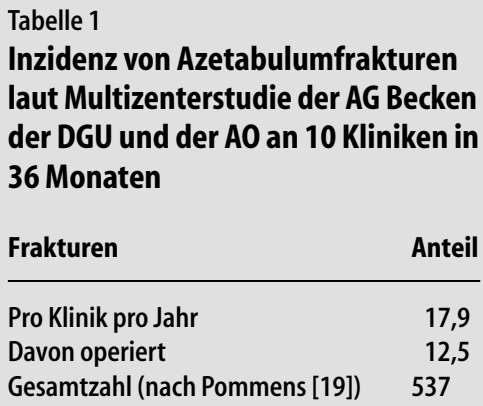

Gesamtzahl (nach Pommens [19])

537

lerfrakturen gehandelt hat, kann der Unfallchirurg außerhalb der Klinik der Maximalversorgung nur auf eine vergleichsweise geringe Zahl von derartigen Rekonstruktionen bei komplexen Azetabulumfrakturen verweisen (Tabelle 1).

Wird weiterhin berücksichtigt, dass allein schon die Zugangswege mit einer nicht geringen Morbidität einhergehen und die Rate postoperativer Komplikationen in Form von Nervenschäden bzw. durch das Auftreten heterotoper Ossifikationen nicht unerheblich ist, muss die Forderung erhoben werden, dass Verletzungen dieser Art nur in ausgewiesenen

(c) Springer-Verlag 2003

Prof.Dr. Kuno Weise

BG-Unfallklinik Tübingen, Schnarrenbergstraße 95, 72076 Tübingen,

E-Mail:weise@bgu-tuebingen.de,

Phone: 07071-6081000, Fax: 07071-6061002 


\section{K. Weise}

\section{Selection of approach and stabilization technique in complex fractures of the acetabulum}

\section{Abstract}

Complex acetabular fractures are defined as fractures involving more than one column of the acetabulum. These fracture types can be categorized with reference to either the Judet and Letournel classification or the AO classification system. For internal fixation of most complex acetabular fractures two different approaches are available: the KocherLangenbeck-approach and the ilioinguinal approach. Which of these nonextensile approaches is selected depends on the type and the localization of the fracture. There are only limited indications for the extensile approaches, because of their considerable morbidity. Several specific tools are available to achieve anatomical reduction. For the stabilisation of complex acetabular fractures lag screws and $3.5-\mathrm{mm}$ reconstruction plates are recommended. The treatment of complex acetabular fractures demands experienced and skilled surgeons; more junior colleagues should be trained in specific operation courses.

\section{Keywords}

Complex acetabular fractures - Selection of approach · Osteosynthesis
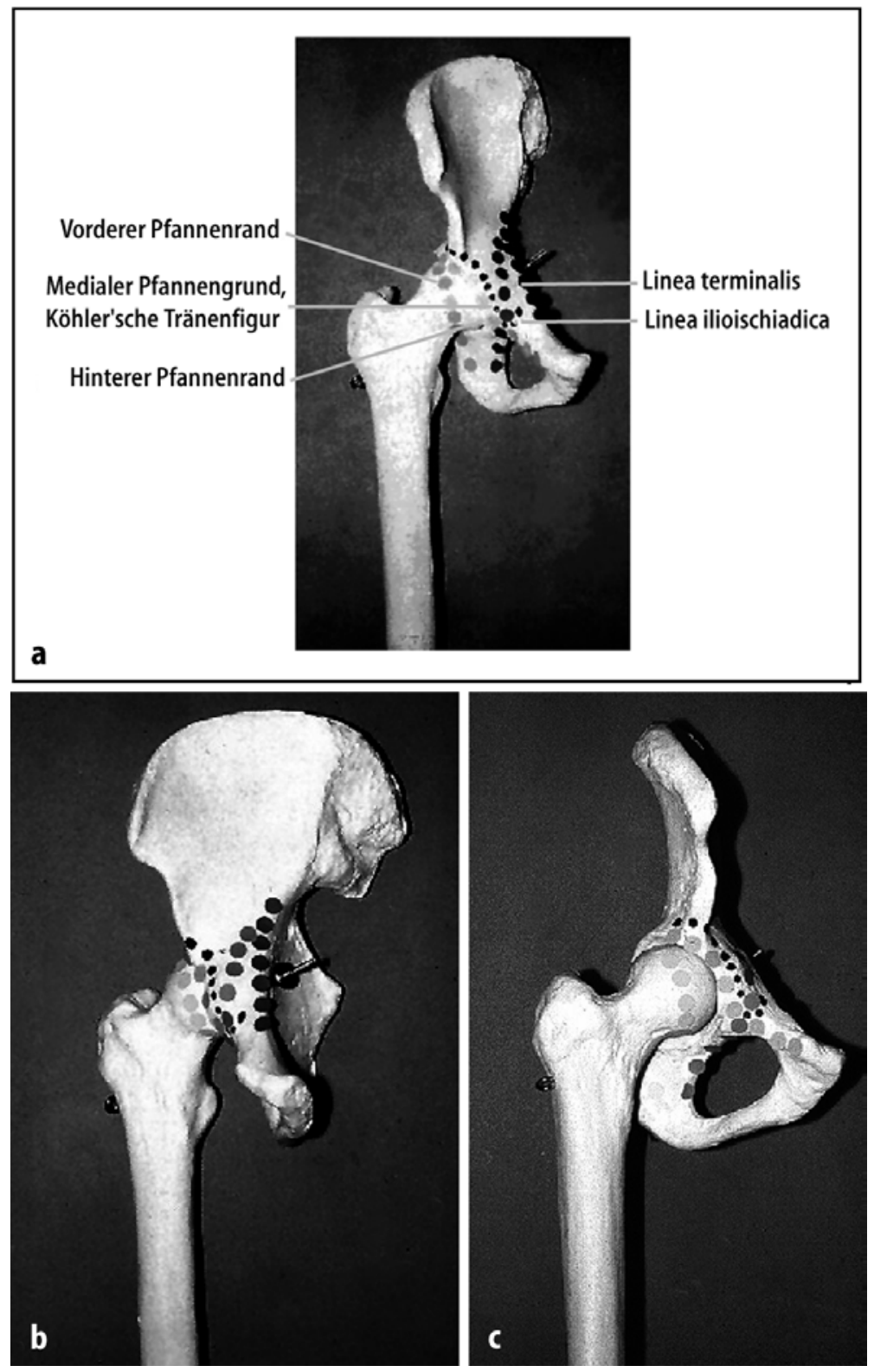

Abb. 1a-c $\boldsymbol{\Delta}$ Leitlinien zur Feindiagnostik von komplexen Azetabulumfrakturen, a a.-p.Projektion des Hüftgelenks mit Markierung der verschiedenen Leitlinien, $b$ Alaaufnahme mit randbildendem vorderem Pfannenrand, $c$ Obturatoraufnahme mit randbildendem hinterem Pfannenrand

und mit der erforderlichen Infrastruktur ausgestatteten Zentren versorgt werden $[13,19,25]$. Gleichwohl sollte die Schulung der sich auf eine umfangreiche Diagnostik stützenden Typisierung und Klassifizierung komplexer Azetabulumfrakturen vorangetrieben und in speziellen Operationskursen an humanen Präparaten die jeweilige Technik der Standardzugänge einschließlich der gebräuchlichen Stabilisierungsverfahren geübt werden.

Rommens u. Hessmann und Rommens et al. [19, 21] publizierten eine ge- schlossene Serie von 225 selbst operativ behandelten Azetabulumfrakturen. Dabei wurde nach dem Score von Merle d'Aubigné in $>85 \%$ der Fälle ein gutes bis sehr gutes Ergebnis nachgewiesen, wobei die Nachuntersuchung bei insgesamt 151 Patienten nach einem Beobachtungsintervall von gut 2 Jahren erfolgte. 189 Patienten wurden über die Standardzugänge nach Kocher-Langenbeck bzw. den ilioinguinalen Zugang [12, 20] versorgt, jeweils $2 / 3$ der Fälle von dorsal, $1 / 3$ von ventral. 

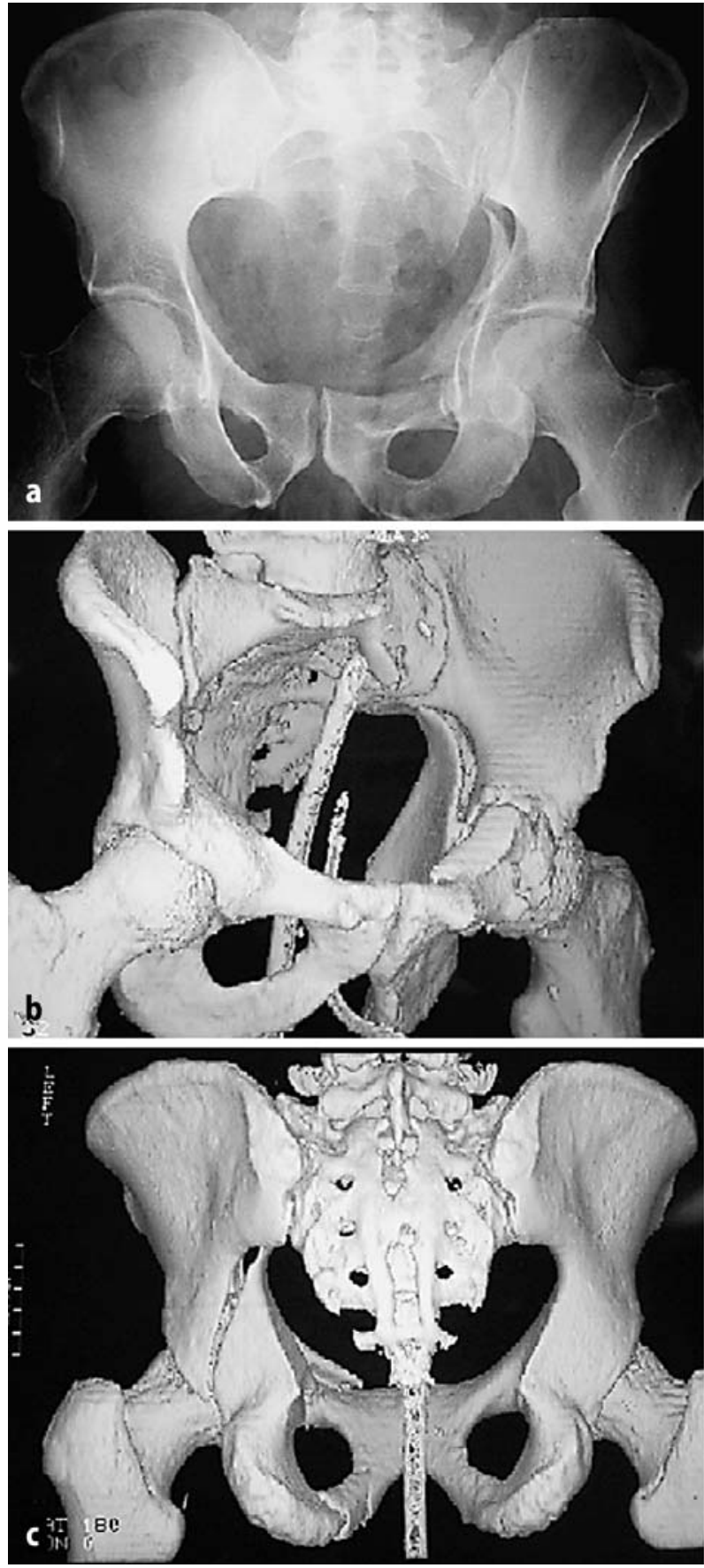
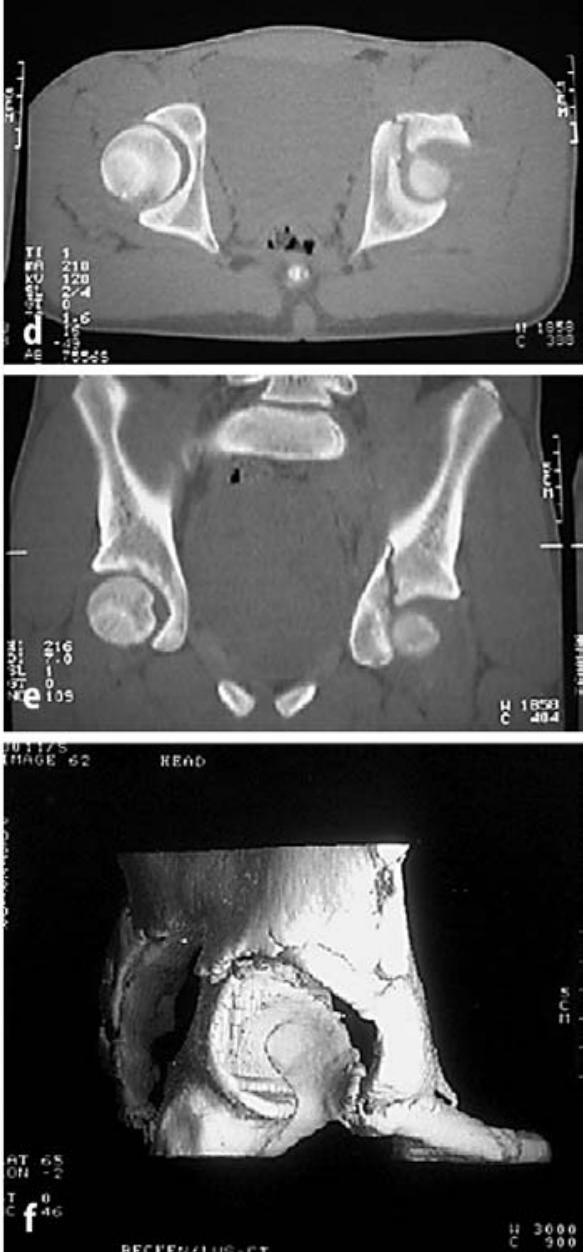

PRAT 64

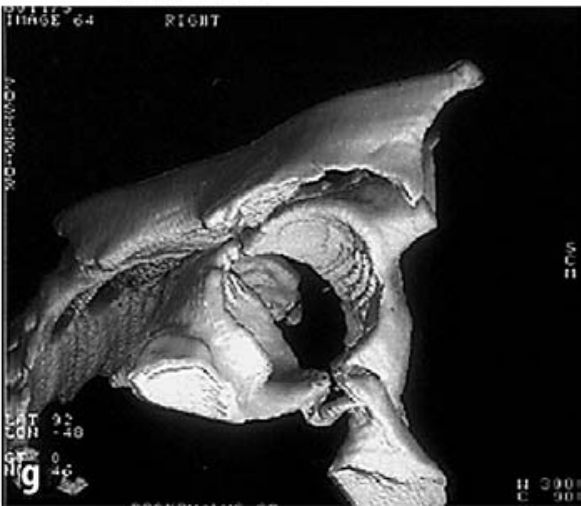

Abb. 2a-g $<$ Möglichkeiten der Bild gebenden Verfahren, a Beckenübersichtsaufnahme, b,c dreidimensionale Rekonstruktion einer 2-Pfeiler-Fraktur mit vorwiegender Betroffenheit des vorderen Pfeilers, d,e zweidimensionale Rekonstruktion, $\mathrm{f}, \mathrm{g}$, herausgerechneter" Hüftkopf mit Beurteilungsmöglichkeit der Hüftpfanne
Größere Serien anderer, in der Azetabulumchirurgie besonders ausgewiesener Autoren aus den 1990er Jahren verwiesen auf ähnliche Resultate, wobei die Rate guter bis sehr guter Ergebnisse zwischen 70 und 90\% schwankte $[8,10$, $13,19]$. Es ist daher zu vermuten, dass die mittel- und längerfristigen Resultate bei weniger erfahrenen Unfallchirurgen unterhalb dieses Levels liegen, da mit einem Ansteigen der bekannten Komplikationen in Form von Nervenläsionen, peri- artikulären Ossifikationen und mehr oder weniger ausgeprägten Arthrosen aufgrund verbliebener Knorpeldefekte und Stufenbildungen der Gelenkfläche gerechnet werden muss $[14,15]$. In diesem Zusammenhang ist festzustellen, dass der Kocher-Langenbeck-Zugang anerkanntermaßen eine höhere Morbidität besitzt als der hinsichtlich der Präparation etwas aufwändigere ilioinguinale Zugang [20].

\section{Diagnostik und Klassifikation}

Unabdingbare Voraussetzung für die Auswahl des geeigneten operativen $\mathrm{Zu}$ gangswegs und die darüber vorzunehmende Art der Stabilisierung bei komplexen Azetabulumfrakturen ist in jedem Fall eine hinreichende Diagnostik. Diese dient als Basis für die Typisierung der Fraktur, aus welcher normalerweise die Art des jeweiligen Vorgehens abgeleitet werden kann. Zur Basisdiagnostik 
zählt die Beckenübersichtsaufnahme einschließlich der axialen Projektion des betroffenen Hüftgelenks, mit Hilfe derer bereits wesentliche Informationen zur Schwere der Verletzung zu gewinnen sind. Ala- und Obturatoraufnahme in Form einer Beckenübersicht mit jeweils um $45^{\circ}$ angehobenem kontra- (Ala-) bzw. ipsilateralem Hüftgelenk (Obturator) lassen vordere und hintere Wand- und Pfeilerfrakturen erkennen, speziell unter Anwendung der so genannten Leitlinien, welche in den verschiedenen Projektionen konturbildend sind (Abb. 1). Während die Alaaufnahme die vordere Pfannenbegrenzung heraushebt und randständig abbildet, werden bei der Obturatoraufnahme der hintere Pfannenrand bzw. Pfeiler „herausgedreht“. Durch „Abfahren" der Leitlinien mit ggf. Feststellung einer Unterbrechung derselben sind Rückschlüsse auf den Frakturverlauf bzw. die Betroffenheit einzelner Bereiche der Hüftpfanne möglich. Hierbei zeigt sich der Vorteil der von Rommens u. Hessmann und Rommens et al. [19, 21] empfohlenen Ala- und Obturatoraufnahme in Form einer Beckenübersicht, da sie einen perfekten Vergleich zwischen verletzter und unverletzter Seite ermöglichen $[1,5,6,7,11,15,18,19,25]$.

Während einige Autoren nur dann ein Computertomogramm für erforderlich halten, wenn Unklarheiten bezüglich der Komplexizität der Verletzung, der Verdacht auf intraartikulär liegende Fragmente oder eine mögliche Schädigung des Hüftkopfs fortbestehen, ist u. E. diese diagnostische Erweiterung nahe$\mathrm{zu}$ ausnahmslos indiziert. Zwei- und dreidimensionale Rekonstruktionen in verschiedenen Schnittebenen und Anund Aufsichten erleichtern die Planung der operativen Strategie einschließlich der Wahl des richtigen Zugangs ungemein. Mit Hilfe von Querschnitten mit sagittaler Rekonstruktion ergeben sich klare Hinweise auf Verlauf, Dislokation bzw. Diastase der Frakturlinien und Fragmente, die Betroffenheit der beiden Pfeiler, nicht zuletzt aber auch auf intraartikulär liegende Fragmente bzw. Frakturen des Hüftkopfs.

Durch „Herausrechnen“ des Hüftkopfs aus der Pfanne gelingt ein ungestörter Einblick in das Azetabulum, sodass das Vorhandensein intraartikulärer Fragmente geklärt und zuverlässige Hinweise zum Frakturverlauf gewonnen werden können $[7,11,18]$ (Abb. 2).
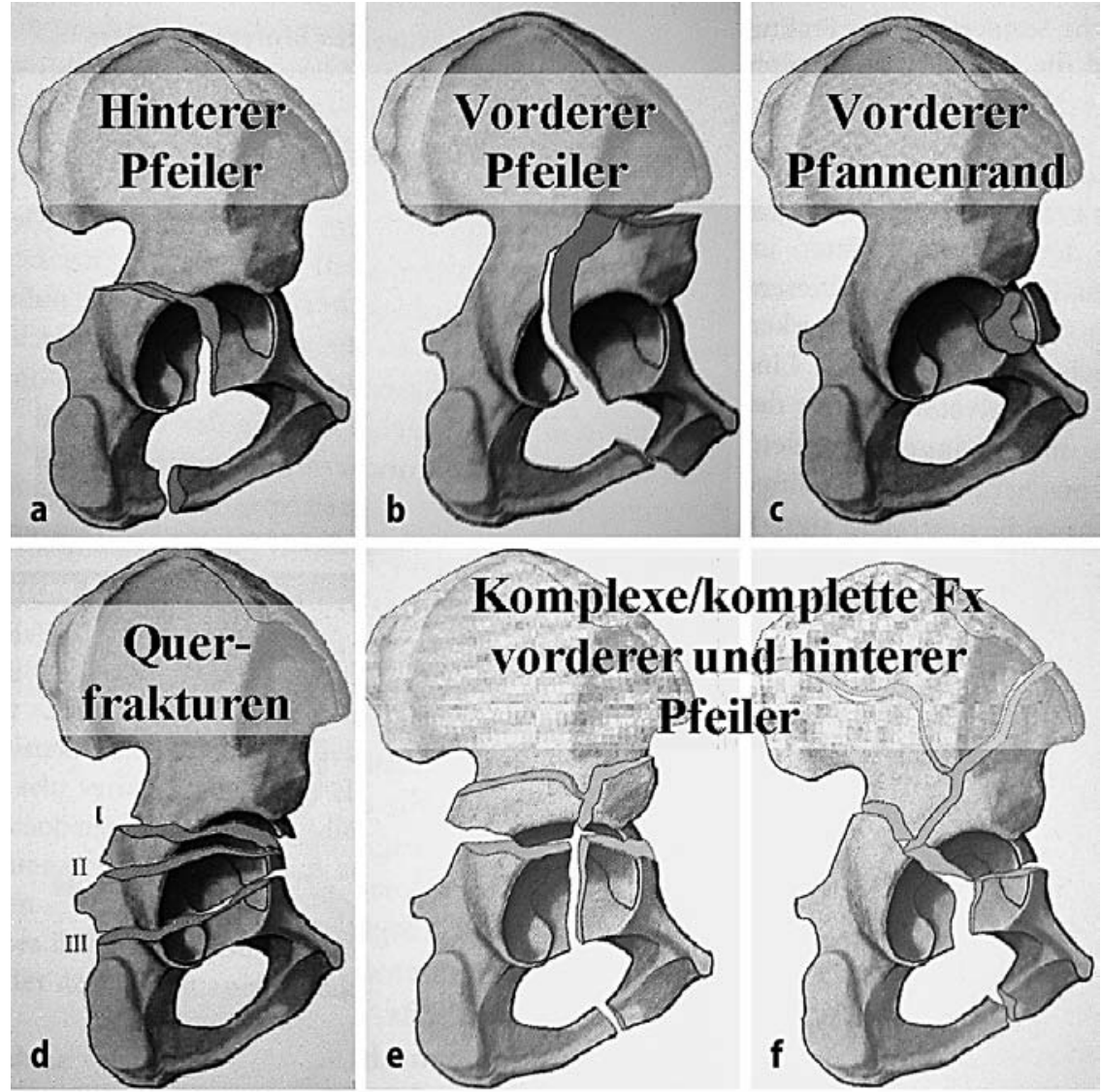

dorsal

ventral

Abb. 3a-f $\boldsymbol{\Delta}$ Klassifikationen, a-c einfache Azetabulumfrakturen nach der Klassifikation von Judet und Letournel, $\mathrm{d}-\mathrm{f}$ komplexe Azetabulumfrakturen

Zur Einteilung der Azetabulumfrakturen hat sich ungeachtet der AOKlassifikation diejenige von Judet und Letournel erhalten, welche so genannte einfache Frakturformen von kombinierten unterscheidet (Abb. 3). Diese Differenzierung lässt aber keine Aussage zur Notwendigkeit des operativen Vorgehens zu, indem auch Frakturen der einfachen Gruppe vielfach eine Indikation zur Rekonstruktion darstellen (z. B. Hinterwandfraktur nach dorsaler Luxation des Hüftkopfs, hohe Querfraktur usw.). Die kombinierten Frakturtypen entsprechen weitgehend den Typen $\mathrm{B}$ und $\mathrm{C}$ der AO-Klassifikation, da hierbei beide Pfeiler involviert sind [10,17] (Abb. 4).

Zur Entscheidungsfindung bezüglich eines möglichen konservativen Vorgehens ist die Beurteilung der Unversehrtheit des so genannten „weightbearing dome" von Interesse, für deren Bestimmung Matta [13] Kriterien der Vermessung angegeben hat $[13,18,19]$.
Durch Einzeichnen einer vertikalen, vom Zentrum des Hüftkopfs nach kranial verlaufenden Linie und einer 2. Linie vom Hüftzentrum zum Schnittpunkt der Fraktur am Pfannenrand, und dies in allen 3 Projektionen, kann gemessen werden, ob eine Stufe $>2 \mathrm{~mm}$ oder ein Winkel $<45^{\circ}$ bestehen, was eine geringe Kongruenz zwischen Hüftkopf und Pfannendach und damit eine Operationsindikation bedeuten würde.

Zusammenfassend sollen Diagnostik und Klassifikation zur Abklärung der Frage nach der geeigneten Therapie (konservativ/operativ?) und für den Fall einer notwendigen operativen Rekonstruktion auch zur Wahl des passenden Zugangswegs beitragen. Wenn immer möglich, sollte die Rekonstruktion einer komplexen Azetabulumfraktur innerhalb der ersten 3-7 Tage nach der Verletzung vorgenommen werden, da dies sowohl die Reposition begünstigt als auch das Risiko bezüglich der heteroto- 


\section{Klassifikation}

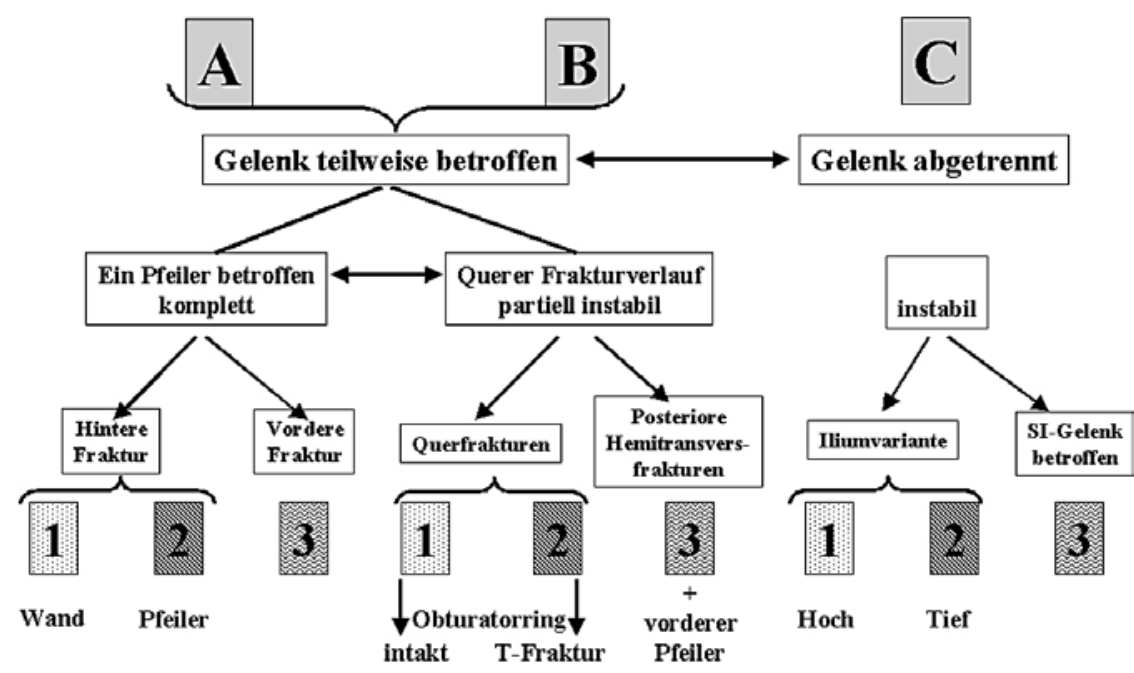

Abb. $4 \Delta$ A0-Klassifikation nach Müller - Komplexe Azetabulumfrakturen Typ B und C

pen Ossifikationen verringert $[6,13,19$, 24].

\section{Zugangswege}

Für die Versorgung nahezu aller Frakturtypen reichen der Kocher-Langenbeck-Zugang oder der ilioinguinale $\mathrm{Zu}$ gang aus. In Fällen komplexer 2-PfeilerFrakturen kann es ausnahmsweise notwendig werden, einzeitig beide Standardzugänge zu verwenden, um die Hüftpfanne rekonstruieren zu können. So genannte erweiterte Zugänge kommen nur in seltenen Fällen bei hohen transtektalen Frakturen bzw. komplexen Verletzungen des Pfannendachs mit dislozierten Brüchen des Os ileum in Betracht [16]. Nach Rommens u. Hessmann [19, 20] können alle Frakturen der Hinterwand und/oder des hinteren Pfeilers über den dorsalen Zugang versorgt werden, wohingegen vordere Pfeilerfraktu- ren und die meisten 2-Pfeiler-Frakturen über den ilioinguinalen Zugang stabilisiert werden sollten. Da die Mehrzahl der Azetabulumfrakturen den dorsalen Wand- bzw. Pfeilerbereich mit oder ohne Beteiligung des kranialen Hüftpfannenabschnitts tangiert, wird überwiegend der dorsale Zugang gewählt. Diese Tatsache lässt sich mehrheitlich auch aus der Publikation größerer Patientenkollektive ersehen, obgleich der ilioinguinale Zugang ungeachtet der höheren Anforderungen an die Präparation wichtiger Strukturen zahlenmäßig an Bedeutung zu gewinnen scheint $[10,20]$.

Mit Hilfe einer Trochanterosteotomie z. B. in Form der so genannten Fliposteotomie gelingt eine deutliche Erweiterung der Übersicht mit verbesserten Chancen, vom dorsalen Zugang aus mehr ventral gelegene Fragmente reponieren und stabilisieren zu können [22] (Abb. 5).
Der hintere Zugang ist durch eine nicht unerhebliche Rate an Schädigungen des $N$. ischiadicus (6-8\% nach der Literatur), eine mögliche Schädigung des glutealen Gefäß-Nerven-Bündels mit konsekutiver muskulärer Insuffizienz und Trendelenburg-Hinken sowie einer nicht geringen Rate bezüglich der Ausbildung heterotoper Ossifikationen belastet. Der ilioinguinale Zugang gefährdet den N. cutaneus femoris lateralis und das Gefäß-Nerven-Bündel in der Leiste. Zudem können lymphatische Abflussstörungen auftreten.

\section{Operationstechnik}

Der Kocher-Langenbeck-Zugang erfolgt meist in Seitlage mit beweglich abgedecktem Bein, wobei eine steril eingepackte suprakondyläre Extension für die Reposition gute Dienste leistet. Das Bein kann auf diese Weise gestreckt und innenrotiert werden, wodurch der N. ischiadicus eine Entlastung erfährt. Einige $\mathrm{Au}$ toren empfehlen die Bauchlage, bei welcher aufgrund der Überstreckung im Hüftgelenk und zusätzlich gefördert durch Kniebeugung der N. ischiadicus noch größeren Schutz genießt. Dieser Zugang hat u. E. den Nachteil, dass die intraoperative Entscheidung zu einem zweiseitigen Vorgehen nur mit höherem Aufwand möglich ist und die Adaption des Operateurs an die Anatomie gewöhnungsbedürftig ist $[6,8,10,15,19]$ (Abb. 5).

Der ilioinguinale Zugang wird in Rückenlage auf einem röntgendurchlässigen Operationstisch ausgeführt. Wenn allein über diesen Zugang eine ausreichende Reposition der hinteren Frakturlinien z. B. bei 2-Pfeiler-Frakturen nicht gelingt, muss intraoperativ umgelagert werden. Bei beiden Zugängen ist darauf zu achten, dass die ohnehin

Abb. 5a-c $\nabla$ Kocher-Langenbeck-Zugang, a Schnittführung in Seitlage, b anatomischer Situs im Schema, c intraoperativer Situs mit Darstellung der kleinen Außenrotatoren
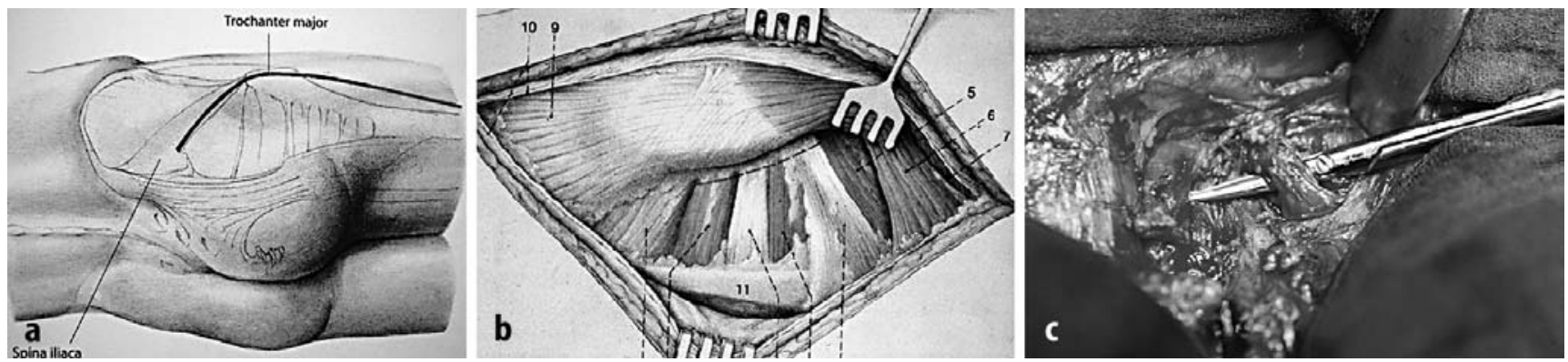

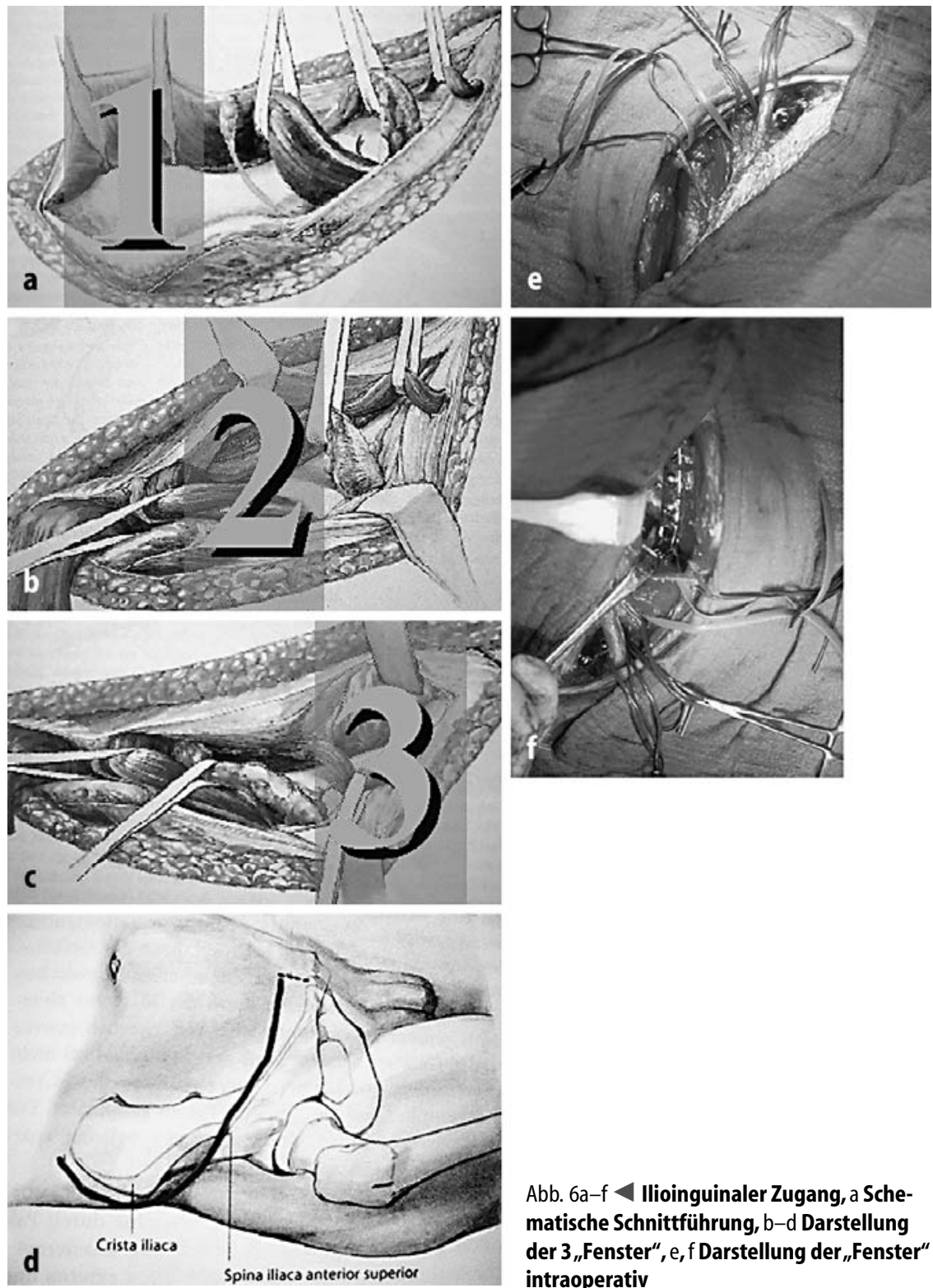

Abb. 6a-f $<$ Ilioinguinaler Zugang, a Schematische Schnittführung, $b-\mathrm{d}$ Darstellung der 3 „Fenster", $e$, f Darstellung der „Fenster" intraoperativ

schwierige intraoperative Durchleuchtung zur Überprüfung des Repositionsergebnisses bzw. der Implantatlage nicht verdeckt ist, was vor Hautdesinfektion und steriler Abdeckung geprüft werden sollte. Diesbezüglich werden C-Bogengestützte Navigationstechniken mit intraoperativ möglicher mehrdimensionaler Bildgebung zukünftig eine erhebliche Erleichterung bewirken [12, 20] (Abb. 6).

Die Reposition deutlich dislozierter komplexer Azetabulumfrakturen ist schwierig und bedarf neben dem Zug am betroffenen Bein durch einen Assistenten zusätzlicher Hilfsmittel. Zur Rekonstruktion hinterer Pfannenanteile können spezielle Zangen verwendet wer- den, welche wie die Jungbluth-Zange teilweise mit Schrauben im supraazetabulären Bereich zu befestigen sind und die Ausübung komprimierender sowie „scherender“ Repositionsschritte ermöglichen. Für uns hat sich für solche Fälle nicht zuletzt der Distraktor sehr gut bewährt, mit welchem eine Subluxation des Hüftkopfs erreicht und die Entfernung intraartikulär gelegener Fragmente vorgenommen werden können. Ein weiteres Hilfsmittel ist die in den absteigenden Sitzbeinast kaudal des gedachten Plattenendes eingebrachte SchanzSchraube, an welcher mittels eines JakobsFutters beträchtlicher Zug ausgeübt werden kann und die gleichzeitig als Weichteilretraktor dient $[6,15,19,25]$ (Abb. 7).
3,5-mm-Rekonstruktionsplatten vorzugsweise aus Titan - eignen sich besonders für die Adaption an den hinteren Pfeiler, da sie gebogen und geschränkt werden können, wobei sich das Biegeplättchen als Modell für die Konturierung eignet. 3,5- bzw. 4,5-mm-Kortikalisschrauben sind in Zugschraubentechnik zur Erzeugung interfragmentärer Kompression von Vorteil, sie werden supraazetabulär zwischen innerer und äußerer Kortikalis des Os ileum platziert (z. B. von dorsal nach ventral). Kleinere Pfannenrandfrakturen können allein mit Zugschrauben fixiert werden, in der Regel wird ein solches Fragment zuverlässiger mit einer kleinen Rekonstruktionsplatte retiniert $[6,19,21,25]$ (Abb. 7c-e).

Die Stabilisierung komplexer Frakturen des vorderen Pfeilers über den ilioinguinalen Zugang wird durch die Präparation der 3 so genannten „Fenster“ ermöglicht, die durch Verlagerung der angeschlungenen Strukturen erweitert werden können. Durch Ausdehnung des Zugangs auf die Innenseite des Os ileum gelangt man bis zur Crista iliaca bzw. zur Sakroiliakalfuge, sodass komplexe Frakturen auch dieser Bereiche von kranial nach kaudal anatomisch rekonstruiert und mit kleinen Plättchen (2- bis 3-LochPlatten) bzw. isolierten Zugschrauben stabilisiert werden können. Entlang der Crista iliaca ist zur Sicherung des korrekten Niveaus der Hauptfragmente des Os ileum das Fixieren einer Drittelrohrplatte möglich. Nach Stabilisierung des Os ileum erfolgt jetzt die Rekonstruktion der Hüftpfanne von vorn nach hinten, wobei der Einblick in das Gelenk von vorn nicht möglich ist und die Entfernung kleiner freier Gelenkkörper allenfalls über den Frakturspalt vorgenommen werden kann. Fallweise müssen lange 3,5-mm-Rekonstruktionsplatten zum Einsatz kommen, wenn die Stabilisierung auf den ipsi- oder gar kontralateralen oberen Schambeinast ausgedehnt werden muss $[6,15,19,25]$ (Abb. 8).

Eine Ausnahme stellen komplexe Azetabulumfrakturen im hohen Lebensalter dar, insbesondere wenn bereits deutliche arthrotische Veränderungen des Hüftgelenks zu verzeichnen sind. Sofern es möglich ist, einen Pfannenabstützring supraazetabular bzw. im oder am absteigenden Sitzbeinast zu verankern, ist der primäre totalendoprotheti- 

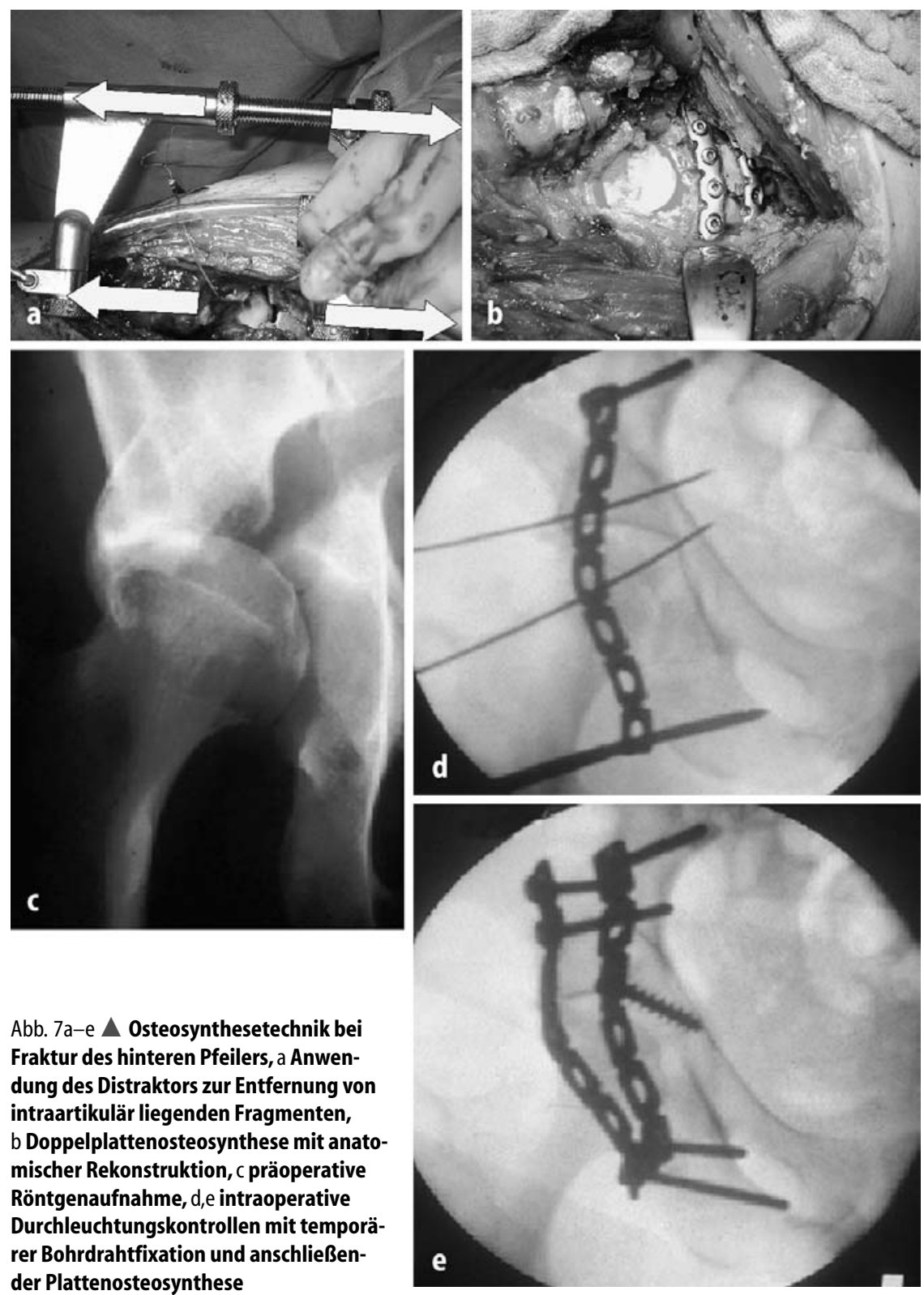

Abb. 7a-e $\Delta$ Osteosynthesetechnik bei Fraktur des hinteren Pfeilers, a Anwendung des Distraktors zur Entfernung von intraartikulär liegenden Fragmenten, b Doppelplattenosteosynthese mit anatomischer Rekonstruktion, c präoperative Röntgenaufnahme, $d, e$ intraoperative Durchleuchtungskontrollen mit temporärer Bohrdrahtfixation und anschließender Plattenosteosynthese

sche Ersatz des Hüftgelenks das Verfahren der Wahl. Autologer Knochen aus dem resezierten Hüftkopf kann zur Unterfütterung des Abstützrings eingebracht werden. Ist dessen sichere Fixation in Frage gestellt, wird eine konservative Therapie mit Extensionsbehandlung und schrittweise vorgenommener Mobilisierung vorangestellt, um nach Abheilen der Fraktur die totalendoprothetische Ersatzoperation durchzuführen. Vorteil dieser Vorgehensweise ist, dass in vielen Fällen ein Abstützring dann nicht mehr benötigt wird, der Nachteil dieser Strategie liegt in der anfänglichen Bettruhe über etwa 4 Wochen $[3,4,9,23]$.
Bezüglich der Begleit- und Nachbehandlung komplexer Azetabulumfrakturen ist individuell vorzugehen, wobei eine postoperative Extensionsbehandlung regelmäßig entbehrlich sein sollte. Frühzeitige Bewegungsübungen sind wichtig, die Mobilisierung geschieht normalerweise unter Teilbelastung der betroffenen Extremität. Wir führen regelmäßig eine Ossifikationsprophylaxe mit einem nichtsteroidalen Antirheumatikum, z. B. Diclofenac oder Ibuprofen, durch. Spätestens nach 10-12 Wochen sollte nach operativer Rekonstruktion selbst komplexer Azetabulumfrakturen Vollbelastung erreicht sein [19, 25].

\section{Diskussion}

Die operative Versorgung komplexer Azetabulumfrakturen gehört $\mathrm{zu}$ den schwierigsten Aufgaben in der Unfallchirurgie und sollte daher erfahrenen und mit solchen Eingriffen vertrauten Chirurgen vorbehalten bleiben [19, 20]. Grundlegende Voraussetzung für eine erfolgreiche Rekonstruktion ausgedehnter knöcherner Verletzungen der Hüftgelenkpfanne ist eine umfangreiche Diagnostik, die von den üblichen Standardprojektionen über Ala- und Obturatoraufnahmen in Form einer Beckenübersicht bis zur computertomographischen Rekonstruktion in zwei- und dreidimensionaler Technik reicht $[1,11,18$, 25]. Mit Hilfe dieser exakten Lokalisation und Typisierung einer komplexen Azetabulumfraktur gelingt es, deren Einordnung in die etablierten Klassifikationsschemen von Judet und Letournel bzw. der AO vorzunehmen [10, 17]. Durch die damit erfolgte Bestimmung des Frakturtyps wird die Zugangswahl maßgeblich beeinflusst, wobei die weitaus größte Zahl der operativen Rekonstruktionen selbst komplexer Bruchformen über die beiden Standardzugänge nach Kocher-Langenbeck bzw. ilioinguinal bewerkstelligt werden kann $[6,12,19$, 20]. In Einzelfällen kann in einer Sitzung ein zweiseitiges Vorgehen gewählt werden, sofern eine ausreichende Rekonstruktion und Fixation über einen $\mathrm{Zu}$ gang nicht gelingt. Die Trochanterosteotomie beim hinteren Zugang stellt eine Möglichkeit dar, in Fällen schwieriger Reposition einen zweiseitigen Zugang $\mathrm{zu}$ umgehen $[15,22]$. So genannte erweiterte, teilweise sehr ausgedehnte $\mathrm{Zu}$ gänge sind hohen transtektalen Frakturtypen und Spätrekonstruktionen vorbehalten $[8,16]$. Alle Zugänge besitzen per se eine mehr oder weniger ausgeprägte Morbidität im Sinn von Vaskularisationsstörungen, Nervenschäden und Weichteilverkalkungen, sodass das sichere Beherrschen der Operationstechnik unabdingbare Voraussetzung für die Durchführung solcher Eingriffe ist $[6,13$, $14,19]$.

Bei der vielfach schwierigen, weil mit hohen Kräften verbundenen Reposition bedarf es des Wissens um mögliche Repositionshilfen wie speziellen Zangen, den Distraktor und SchanzSchrauben. Ebenso wichtig ist die Reihenfolge der Reposition von kranial 


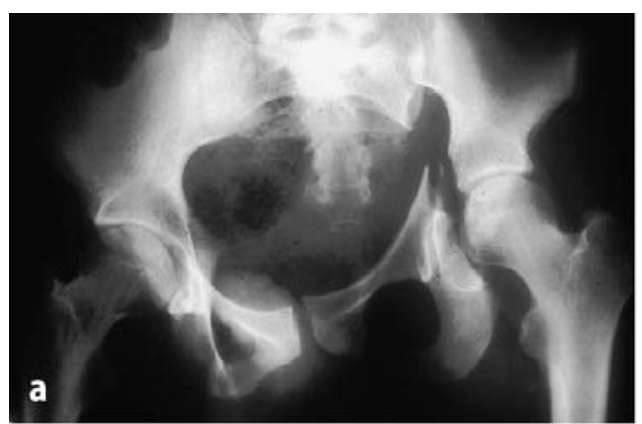

Abb. 8a- $f<$ Versorgung einer Fraktur des ventralen

Pfeilers, a Unfallbild, b-e dreidimensionale Rekonstruk-

tionen aus verschiedenen Blickwinkeln, $f$ Plattenosteo-

synthese bis zum oberen Schambeinast
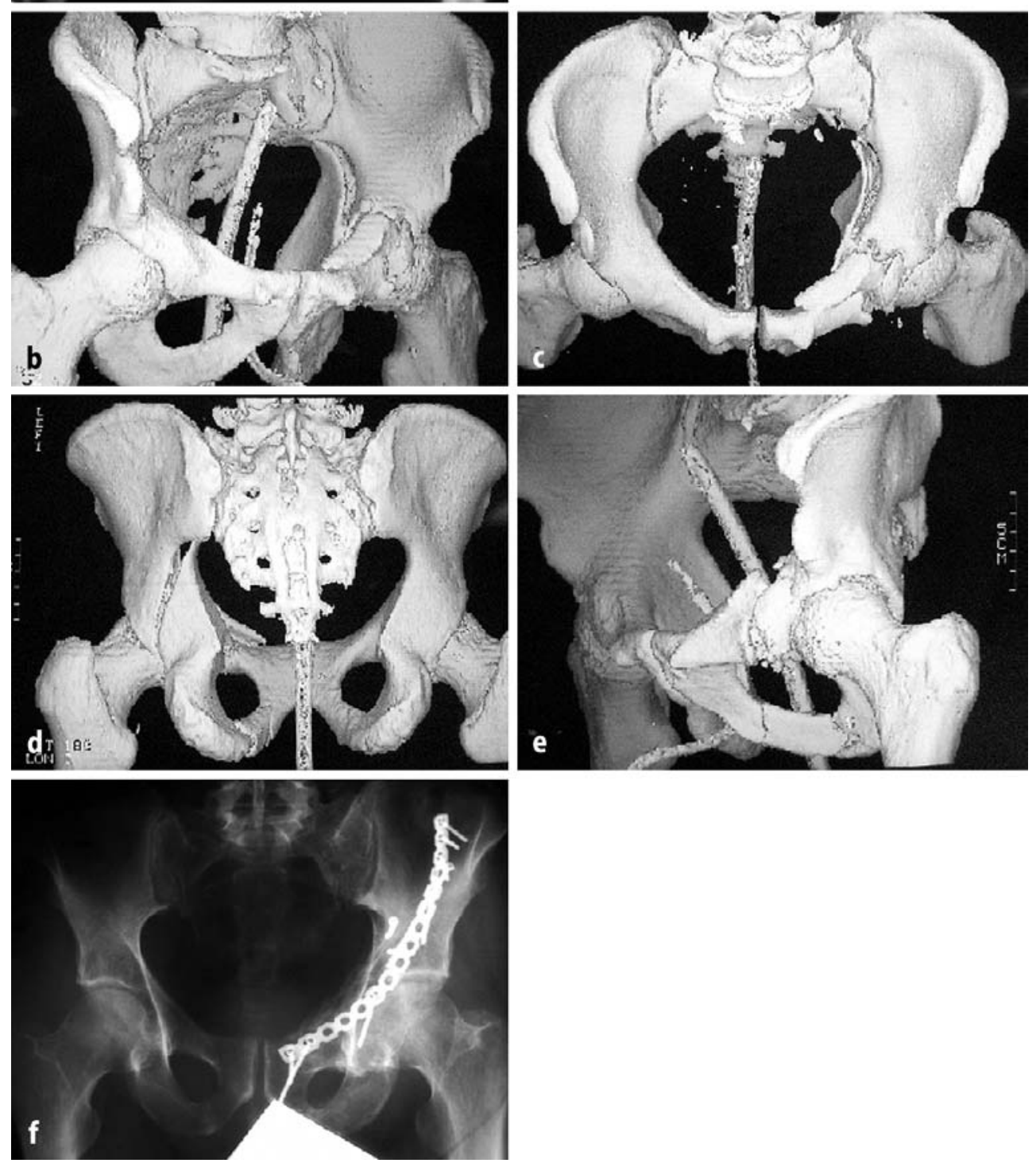

nach kaudal bzw. von ventral nach dorsal beim ilioinguinalen Zugang. Die Fixation erfolgt in der Regel mit Implantaten aus dem Kleinfragmentinstrumentarium, in besonderen Situationen mit den den anatomischen Verhältnissen am besten anzupassenden 3,5-mmRekonstruktionsplatten $[6,13,15,21,25]$.

Ungeachtet der korrekten Reposition und zuverlässigen Retention heilen komplexe Azetabulumfrakturen in einem nicht geringen Prozentsatz mit Sekundärschäden wie einer posttraumatischen Arthrose, einer Hüftkopfnekrose oder einer aufgrund von Ossifikationen beeinträchtigten Funktion aus. Diese Tatsache ist einmal mehr Hinweis darauf, dass die Versorgung komplexer Verletzungen zum frühestmöglichen Zeitpunkt nach umfangreicher Diagnostik und exakter Planung durch einen in der Hüftchirurgie sehr erfahrenen Operateur vorgenommen werden sollte, um die in der Literatur angegebenen Ergebnisse zu erreichen. In Fällen einer ausgedehnten Zertrümmerung der Hüftpfanne kann mitunter ein konservatives Therapieregime bessere Alternativen bieten. Nach Glombik u. Muhr [6] stellen Entscheidungskriterien und Erfolgs- 
aussichten auf 6 wesentliche Kriterien $\mathrm{ab}$ :

1. den Frakturtyp

2. das Ausmaß an Dislokation

3. den Ort der Dislokation („,je höher die Fraktur, desto schlechter die Prognose")

4. die Erfahrung des Chirurgen

5. das Alter des Patienten

6. die Art bzw. den Umfang der Begleitverletzungen

Angesichts dieser Parameter ist es ganz selbstverständlich, die Forderung nach Optimierung und Intensivierung einer speziellen Schulung in Diagnostik und Operationstechnik derartiger Verletzungen zu erheben, welche speziell auf Operationskurse an humanen Präparaten abhebt. Dennoch bilden komplexe Azetabulumfrakturen eine Verletzungsentität, welche im Fall der notwendigen operativen Rekonstruktion eine originäre Aufgabe für unfallchirurgische Zentren und in der Hüftchirurgie ausgewiesene Operateure darstellt.

\section{Literatur}

1. Ambacher T, Kälicke T, Muhr G, Arens S (2000) Klassifikation und Diagnostik von Acetabulumfrakturen. Trauma Berufskrankh 2: 19-27

2. Arbeitsgruppe Becken (AO/DGU) (1997) Beckenringverletzungen/Pelvic injuries. Ergebnisse einer prospektiven, multizentrischen Studie. Hefte Unfallchir 266

3. Baumgärtel F, Feld C, Bohnen L, Gotzen L (1994) Acetabulumfrakturen im höheren Lebensalter. In: Ramanzadeh R (Hrsg) Unfallund Wiederherstellungschirurgie des proximalen Femurs und des Beckengürtels. Einhorn, Schwäbisch Gmünd, S 98-100

4. Berner M, Ulrich C (1999) Die primäre endoprothetische Versorgung von Acetabulumfrakturen. Osteosynthese Int 7: 93-100

5. Fenzl G, Fischer G, Galle P (1990) Acetabulumfrakturen - operative versus konservative Behandlung. Unfallchirurgie 16:230-235

6. Glombik T,Muhr G (2000) Behandlungsstrategie bei Acetabulumfrakturen. Trauma Berufskrankh 2: 46-59

7. Heeg M, Oostvogel HJM, Klasen HJ (1987) Conservative treatment of acetabular fractures: the role of the weight-bearing dome and anatomic reduction in the ultimate results. JTrauma 27: 555-559

8. Helfet DL, Borelli J, DiPasquale T, Sanders R (1992) Stabilisation of acetabular fractures in elderly patients.J Bone Joint Surg Am 74: 753 765

9. Hoellen IP, Mentzel M, Bischoff M, KinzI L (1997) Acetabulumfrakturen beim alten Menschen. Primäre endoprothetische Versorgung. Orthopäde 26: 348-353

10. Judet $R$, Letournel $E$ (1964) Fractures of the acetabulum: classification and surgical approaches for open reduction.J Bone Joint Surg Am 46: 1615-1646

11. Kreitner KF, Rommens PM (1999) Radiologische Diagnostik von Becken- und Acetabulumfrakturen. 0steosynthese Int 9: 3-12

12. Matta JM (1994) Operative treatment of acetabular fractures through the ilioinguinal approach. A 10-year perspective. Clin Orthop 305: 10-19
13. Matta JM (1996) Fractures of the acetabulum. Accuracy of reduction and clinical results in patients managed within three weeks after the injury.J Bone Joint Surg Am 78: 1632-1645

14. Matta JM, Siebenrock KA (1997) Does indomethacin reduce heterotopic bone formation after operations for acetabular fractures? A prospective randomised study.J Bone Joint Surg Br 79: 959-963

15. Maurer F, Volkmann R, Weise K (1993) Verletzungen des Acetabulums. OP J 1: 20-25

16. Mears D, Rubash H (1983) Extensile exposure of the pelvis. Contemp Orthop 6:21-31

17. Müller M (1996) The comprehensive classification of fractures. Part 2: Pelvis and acetabulum. Springer, Berlin Heidelberg New York

18. Olson SA, Matta JM (1993) The computerized tomography subchondral arc: a new method of assessing acetabular articular continuity after fracture (a preliminary report).J Orthop Trauma 7: 402-413

19. Rommens PM, Hessmann MH (1999) Acetabulumfrakturen. Unfallchirurg 102: 591-610

20. Rommens PM, Hessmann MH (1999) Der ilioinguinale Zugang. Osteosynthese Int 9: 74-83

21. Rommens PM, Broos PL, Vanderschot P (1997) Vorbereitung und Technik der operativen Behandlung von 225 Acetabulumfrakturen. Unfallchirurg 100: 338-348

22. Siebenrock KA, Gautier E, Ziran BH, Ganz R (1998) Trochanteric flip osteotomy for cranial extension and muscle protection in acetabular fracture fixation using a Kocher-Langenbeck approach.J Orthop Trauma 12: 387-391

23. Volkmann R, Maurer F, Eingartner C, Weller $S$ (1995) Primär totalendoprothetischer Hüftgelenkersatz bei Acetabulumfrakturen. Unfallchirurgie 21: 292-297

24. Weise K, Weller S (1987) Die konservative Therapie beim Hüftpfannenbruch - Indikation und Ergebnisse. Aktuelle Traumatol 17: 277-283

25. Weise K, Maurer F, Schrade J (1997) Hüftgelenkluxationen und -frakturen, Acetabulumfrakturen. Orthopäde 26: 336-374 